\title{
La Medicina Familiar y Comunitaria en Uruguay de 1997 a 2019: ¿cuántos kilómetros faltarán para llegar al pueblo aquel?
}

\author{
Family and Community Medicine in Uruguay from 1997 to 2019: \\ how many kilometers will it take to reach that town?
}

Jacqueline Ponzo (https://orcid.org/0000-0002-8329-7430) ${ }^{1}$

${ }^{1}$ Facultad de Medicina, Universidad de la República. Av. General Flores 2125.

11.800 Montevideo

Departamento de

Montevideo Uruguai.

ponzo4@gmail.com

\begin{abstract}
The Family and Community Medicine Residency started in Uruguay in 1997. Through a self-managed process, the first generations were molded into training that integrated hospital knowledge and experience with territorial praxis in a community-based health service with a population of reference. The academic recognition of the specialty and the installation of the institutional areas for its management were achievements parallel to that process in the first decade. The second decade was marked by the territorial teaching-assistance expansion in the country, university decentralization and the active participation of Family and Community Medicine in the Health Reform, and the country's rights agen$d a$. The third decade of the specialty begins with a crisis triggered by the sustained decline in the aspiration for residency. An initial approach to explanations reflects on the possibility of facing a more profound crisis and the need to find the keys to a 21st century Medicine that allows us to achieve the principles of Alma-Ata that are still current. Key words Family and community medicine, Staff development, Primary Health Care, Workforce
\end{abstract}

Resumen La residencia de medicina familiar y comunitaria comenzó en Uruguay en el año 1997. A través de un proceso autogestionado, las primeras generaciones se moldearon en una formación que integraba en ellos el conocimiento y la experiencia hospitalarios junto con la praxis territorial en un servicio de salud de base comunitaria con población de referencia. El reconocimiento académico de la especialidad y la instalación de los ámbitos institucionales para su gestión fueron conquistas paralelas a ese proceso en la primera década. La segunda década estuvo marcada por la expansión territorial de la estructura docente-asistencial, la descentralización de la universidad y la participación activa de la medicina familiar y comunitaria en la reforma de la salud y la agenda de derechos. La tercera década de la especialidad se presenta en su inicio como crisis dada por la caída sostenida en la aspiración a la residencia. Desde una aproximación inicial a las explicaciones, se reflexiona sobre la posibilidad de estar frente a una crisis más profunda y la necesidad de encontrar las claves de una medicina del siglo XXI que permita alcanzar los principios de Alma Ata, siempre vigentes.

Palabras clave Medicina familiar y comunitaria, Desarrollo de personal, Atención Primaria de Salud, Recursos humanos 


\section{Introducción}

La condición de integrante de la primera generación de residentes de medicina familiar y comunitaria (mfyc) en el Uruguay, así como haber permanecido unida a la formación de especialistas desde aquel temprano y postrimero a la vez año 1997 (residente, luego tutora, después docente), permite el intento de contar la historia "por dentro”. Siendo éste un artículo de opinión, la subjetividad muchas veces no admitida por la ciencia, podrá deslizarse con permiso, buscando aportar una perspectiva que en los procesos complejos suele ser difícil encontrar si no es desde allí.

El final del siglo XX donde se inscribió el inicio de la residencia en Uruguay (la última ficha que faltaba al mapa de Sudamérica para completar el puzle de la especialización en medicina familiar/mfyc), fue un tiempo especial. No tanto por los presagios místicos o astrológicos para el cambio de milenio, sino por los procesos vertiginosos en el desarrollo de la informática e internet que -de la mano de Gates y Jobs- cambiarían drásticamente al mundo en las décadas siguientes.

Transcurridos algo más de 20 años desde entonces, desacomodados y vueltos a acomodar luego de los cambios, se puede intuir una brisa similar en este nuevo cambio de década donde avances tecnológicos, desde la robótica y la biotecnología, entre otros, nuevamente van a sacudir el escenario. Sabemos que llegarán cambios, no sabemos aún cuánto nos cambiarán.

También pulsan amenazas por el cambio climático, y crece la tensión que el modelo económico introduce en todos los procesos de la vida. Hechos tangibles con repercusiones sociales, políticas y sanitarias afectan un subcontinente extraordinariamente rico en cultura y naturaleza, cada vez más avasalladas éstas por el extractivismo que desconoce el valor de la vida en favor de las ganancias.

Alejandro Rojas, agrónomo y pedagogo chileno, trabajó junto a Paulo Freire desde la Federación de Estudiantes Universitarios en las campañas de alfabetización del gobierno de Allende. Defensor de la agroecología y la producción a escala humana, Rojas introdujo el concepto de "policultivos de la mente". Se apoya en la idea original de Vandana Shiva que llama "monocultivos de la mente ${ }^{1}$ " a la forma de investigar y al conocimiento producido en ambientes controlados. En contraste con esa forma de conocimiento moldeado por el proyecto cultural del Norte, Rojas describe el conocimiento producido a escala local de la siguiente manera:
"Los campesinos requieren de un conocimiento detallado y profundo de su ecosistema. Están enraizados en sus lugares físicos, lo que los equipa con un tipo de conocimiento involucrado y participativo, en vez del conocimiento desarraigado y remoto que muchas veces resulta de la práctica científica de la agricultura industrial.

Valorizan positivamente las experiencias personales y las creencias como recursos de conocimiento en vez de verlas como un obstáculo para entender el mundo. Se trata de un conocimiento local que se centra en los intereses de la comunidad local ${ }^{2 "}$.

La medicina familiar y comunitaria en Uruguay se ha forjado cual "policultivo de la mente". A partir de experiencias singulares en las que no faltó el profundo conocimiento del contexto y el involucramiento de sus participantes se llega a un resultado totalmente compatible con la descripción de policultivos que hace Rojas: "conocimiento que intenta reproducir y asimilar la complejidad e incertidumbre de la vida, acompañado de procesos de aprendizaje que inspiren la apertura de la mente individual y colectiva de una comunidad de aprendizaje a través de objetivos viables y tangibles ${ }^{2}$."

Este texto está producido desde la experiencia vivida y la reflexión. Los documentos producidos a lo largo de este tiempo se encuentran citados, pero es escaso aún lo que se encuentra documentado de una experiencia que por intensa no ha dejado tiempo suficiente para escribirla. En ese sentido, este artículo contribuye a saldar parte de esa deuda.

\section{Espacios para aprender}

En el año 2000 tuvo lugar en Montevideo el IV Congreso de Medicina Familiar y la III Jornada de Residentes del Cono Sur. El primer año del nuevo milenio era también el del egreso de la primera generación de residentes. Aquel evento tuvo lugar en un clima de incertidumbre. Se contaba con un Programa de residencias completamente formalizado por la Comisión Técnica Nacional integrada por la Facultad de Medicina y el Ministerio de Salud Pública. Allí, a partir de la propuesta de un cirujano, entonces presidente de la Comisión, y la confluencia de voluntades que facilitó los votos para la decisión, se había tomado la iniciativa de abrir la residencia de medicina familiar y comunitaria aún cuando la Facultad (representada en dicha Comisión) no llegaba a acordar su aceptación. 
En Uruguay los títulos profesionales de grado y posgrado son administrados y certificados por las Universidades, no por colegios ni asociaciones profesionales como ocurre en muchos países. En la Universidad de la República (que ocupa más del $90 \%$ de la formación médica en el país en todas sus ramas y niveles) la competencia en materia de especialidades médicas está en la órbita de la Escuela de Graduados dependiente de la Facultad de Medicina.

Para entonces la Escuela de Graduados no solo desconocía el proceso iniciado con la residencia de medicina familiar y comunitaria, sino que tenía una postura contraria a la especialidad. La materialidad de cuatro profesionales egresados de la residencia el 31 de marzo del año 2000 contrastaba con las aseveraciones de la Escuela de Graduados negando la existencia ${ }^{3}$.

Si bien esta negación de la que fuimos objeto durante varios años fue motivo de lucha $a^{4,5}$, nunca tuvimos duda de que la historia iría en el sentido esperado y que llegaríamos "más temprano que tarde" a la obtención del título como especialistas y -lo fundamental- al reconocimiento de la especialidad por la Universidad de la República, lo cual representaría la legitimación definitiva de la medicina familiar y comunitaria en el país.

Lo que verdaderamente nos preocupaba en aquel momento eran "los espacios para aprender". Uruguay instaló el Sistema Nacional Integrado de Salud a partir del año 2008. La organización sanitaria antes de la reforma era fragmentada; había múltiples efectores públicos y privados mal coordinados. Los recursos se concentraban a nivel hospitalario y allí tenía lugar la formación de grado y posgrado de los profesionales de la salud. El primer nivel de atención era escaso, poco jerarquizado, poco profesionalizado; deficitario incluso en la Administración de Servicios de Salud del Estado (ASSE) a pesar de que contaba con una histórica red de policlínicas y centros de salud en todo el país.

La necesidad de desarrollo del primer nivel era evidente. Desde la mirada de los residentes, ese desarrollo era necesario no solo para mejorar el sistema de atención, sino como "espacio para aprender". El asunto estaba presente como necesidad, como boceto de un ideal a alcanzar, como anhelo. La residencia se había puesto a andar sobre los únicos andariveles posibles entonces: por un lado, el hospital, y por otro los tutores ${ }^{6}$. Como tutores fueron designados, por la Comisión Nacional de Residencias, reconocidos médicos y médicas que, sin contar con el título de especialistas (hecho no posible en aquel Uruguay), tenían una práctica excepcional, fundada en los principios de la medicina familiar y comunitaria, establecida en un servicio de salud del primer nivel, con una población de referencia ${ }^{7,8}$.

En el hospital la referencia fueron las Clínicas de pediatría, ginecoobstetricia y medicina interna, los Servicios ambulatorios de algunas especialidades focales como oftalmología, otorrinolaringología y dermatología y los Departamentos de emergencia. Para las y los Profesores a cargo de estos servicios la primera noticia de la residencia de mfyc fue nuestra llegada el día que teníamos que comenzar la pasantía. Llegábamos con el Programa de dos páginas que indicaba simplemente que debíamos estar allí. Informar de qué trataba la "nueva" especialidad era tarea de cada residente; los objetivos e implementación de la pasantía era algo que había que construir junto a los anfitriones a partir de ese momento. No estaba pautado eso en sitio alguno, pero en ese momento surgía claro para residentes y docentes que no había otro camino.

Si bien la actividad hospitalaria comenzó a ser cuestionada como espacio de formación para una especialidad destinada al ejercicio en el ámbito comunitario (en aquel tiempo representaba entre un 90 y $70 \%$ de las 48 horas semanales, decreciente a lo largo de tres años de la residencia), para los residentes de las primeras cohortes no constituía un problema. La recepción había sido muy buena y la posibilidad de contar con docentes calificados fuera del hospital era muy limitada. Además, la experiencia de compartir el aprendizaje en la sala de internación o de urgencias trabajando a la par con los residentes de pediatría, ginecología, medicina interna, cirugía, psiquiatría, entre otros, constituyó un camino de reconocimiento mutuo que fundó bases sólidas para la aceptación de la especialidad en el mundo de la medicina de entonces (dominantemente hospitalario). Nos sentíamos capaces de hacer las "traducciones" necesarias para llevar el aprendizaje hospitalario al ámbito comunitario, y la actividad regular con tutor cumplía un rol fundamental en ese proceso.

No estábamos desconformes con nuestra residencia autodidacta y autogestionada. Esa condición de aparente precariedad fue un valor en sí, e hizo del proceso formativo un camino de liderazgo para la construcción y el posicionamiento de la especialidad. Pero sabíamos que esas condiciones no constituían el ideal. Sabíamos de la necesidad de espacios para aprender, que pudieran acoger a los residentes en un lugar del sistema de salud más cercano a la gente que lo que represen- 
ta el hospital, y que permitieran el desarrollo de todos aquellos procesos que ya sabíamos formaban parte de la especialidad y, por tanto, debían ser parte del camino de formación.

Hablábamos de espacios para lo asistencial, la investigación, el abordaje familiar, el componente comunitario. Identificábamos necesario:

Espacios para la atención: Servicios de salud organizados para la labor asistencial en consultorio y también en domicilio, capaces de mantener una adecuada coordinación con el hospital y los servicios especializados de referencia para los pacientes, ya fuese mediante cartas, visitas, acompañamiento de los pacientes por los residentes.

Espacios equipados: Servicios de salud con recursos e insumos adecuados y suficientes para resolver los principales motivos de consulta de la población de todas las edades, incluso en situaciones de urgencia y emergencia, máxime cuando los servicios de salud se ubicaban en áreas rurales.

Espacios adecuados al modelo de medicina familiar y comunitaria: Con los recursos necesarios para la antropometría de niños y adultos, con camillas ginecológicas y comunes, con posibilidad de atender la población de todas las edades y recibir en la consulta a varios integrantes de una familia.

Espacios para estudiar: Contar con acceso a los libros, las revistas, la bibliografía actualizada dentro de los espacios de labor asistencial, así como con el tiempo y la organización necesarios para la lectura como parte del trabajo cotidiano, a partir de las dudas que instala la clínica, o para sesiones programadas de lectura grupal.

Espacios de calidad, con rigurosidad en procesos y registros. Especial énfasis en el uso de las historias clínicas, preferentemente en formato familiar y el uso del familiograma.

Espacios para investigar: Registros clínicos de calidad, principal insumo para la investigación clínica en el primer nivel de atención. Aproximación epidemiológica a la comunidad para conocer mejor sus necesidades y problemas.

Espacios comunitarios: Acceso a salas de reuniones, tiempo para actividades con la comunidad y desarrollo de estrategias grupales.

Aproximadamente ésta fue la descripción de los "espacios para aprender", dibujada desde la experiencia de residentes, presentada durante el Congreso del año $2000^{9}$. Nunca publicado, este pensamiento colectivo ha sido guía en las trayectorias recorridas en las dos décadas siguientes.

\section{La dialéctica de los inicios}

Una sensación que prevalecía en el/la residente de entonces era la de "procesador". Había mundos de necesidades (bocetos ideales) y mundos de posibilidades (realidades imperfectas). Nada venía hecho, pero nos era dada la posibilidad de construir desde y para la medida de cada uno, de cada una. Sin recetas (el Programa de la residencia se limitaba a aquellas dos páginas que pautaban una lista de rotaciones obligatorias y la carga horaria), lo que había era la experiencia vital y formativa de cada residente con su tutor, y sus trayectorias; desde el domicilio rural a la sala de partos, desde las mañanas de hospital y noches de urgencias a las tardes con niños en las escuelas.

La experiencia iba enriqueciendo las ideas, los bocetos iniciales, y arrojaba insumos para perfeccionar aquel modelo de residencia "ideal" donde soñábamos con un Programa estructurado, docentes propios de la especialidad y ámbitos académicos reconocidos en el primer nivel.

La interacción dialéctica con los hechos cotidianos, el contexto de libertad que ofrecía la flexibilidad del programa, y la constante producción de nuevos hechos y resultados a la medida de las necesidades, era la que nos hacía sentir como "procesadores" vivos: los procesos vividos nos transformaban, pero también nosotros transformábamos, y todo ocurría en una escala individual/local que se amplificaba a medida que las experiencias se integraban y trascendían al nivel institucional que alcanzaba incluso escala nacional.

En la retrospectiva de aquel tiempo, que desde una perspectiva curricular puede resultar pobre (un programa de dos páginas), debe rescatarse la riqueza de la producción (de ideas, de hechos, de cambios), así como la solidez de la formación alcanzada a través de esos procesos caóticos, pero productivos en la medida que uno se ofreciera como "procesador" para dejarse transformar por ellos; lo cual no era tan inocente, pues eso mismo era lo que permitía transformar, sabíamos ${ }^{10}$.

\section{Las unidades docente asistenciales}

Los años 2007 y 2008 fueron intensamente legislativos en Uruguay. Se produjeron normas muy relevantes para la salud. De 2007 es la Ley $18.211^{11}$, de creación del Sistema Nacional Integrado de Salud, que da paso a la reforma sanitaria más importante de la historia del país. Luego, la Ley de Rendición de Cuentas aprobada en 2008 da lugar a la creación del "Programa para 
la Formación y Fortalecimiento de los Recursos Humanos de los Prestadores Públicos de Servicios de Salud" (PFRHS) ${ }^{12}$. En esta Ley se abre un nuevo renglón presupuestal que asigna recursos para su gestión conjunta por la Facultad de Medicina (Universidad de la República) y la Administración de Servicios de Salud del Estado (ASSE) (prestador estatal del SNIS que alberga la mayor parte de la formación de los profesionales de la salud en el país). A partir de esta asignación de fondos se abre la posibilidad de creación de Unidades Docente Asistenciales, prioritariamente en el primer nivel de atención. Se convoca a la postulación de proyectos y equipos con base territorial. Trece proyectos aprobados en el año 2008 ubicados en seis de los diecinueve departamentos del país fueron el inicio de este programa. Quedaron instalados en abril de 2010. En una segunda etapa se incorporaron nuevos proyectos rurales lo cual amplió la cobertura a diez departamentos.

El PFRHS estableció criterios para la selección de los proyectos como forma de promover algunos de los objetivos del programa: la concentración del trabajo, la permanencia en el cargo y la estabilidad de los equipos, así como la protección de la carrera docente y la cooperación en la labor de enseñanza por profesionales sin cargo docente pero avalados por la Facultad.

Aquel boceto del año 2000 se puede descubrir presente, diez años más tarde, en la matriz de organización adoptada por las Unidades Docente Asistenciales del primer nivel.

En diferentes lugares del mapa se fueron radicando equipos con número e integración diversa, predominante pero no exclusivamente de medicina familiar y comunitaria (también integran los equipos pediatras, obstetras, psicólogos, psiquiatras, epidemiólogos, nutricionistas, ginecólogos) dando lugar a un nuevo proceso de construcción dialéctica tan productivo como el de inicio de la residencia. Fue a través de este proceso que aquellos "espacios para aprender" se fueron materializando en el territorio de una forma amplia, superando la fragmentación presente en muchos servicios del primer nivel, para instalar lógicas de proceso, trabajo en equipo y abordaje integral, longitudinal y continuo.

Estos nuevos espacios, constituidos por servicios de salud y sus territorios, pasaron a ser los ámbitos de referencia curricular para la totalidad de los residentes de medicina familiar y comunitaria.

El Departamento de Medicina Familiar y Comunitaria, tras un proceso lento desde el "negacionismo" inicial de la Facultad de Medicina en el
2000 y años previos, había completado en 2007 su proceso de consolidación institucional. En el año 2010 avanza de modo sustantivo a su desarrollo descentralizado, con una base académico-administrativa central, y una amplia red territorial de Unidades Docente Asistenciales donde se produce la totalidad de la actividad asistencial y de enseñanza-aprendizaje clínica y comunitaria, tanto para estudiantes de grado como de posgrado de la especialidad.

Los diez años que nos separan de aquel segundo momento fundacional han sido de trabajo intenso, de desarrollos locales, de maduración y creación, pero también, más recientemente, de crisis. De esto hablaremos más adelante.

\section{Profundización en otro milenio}

En una matriz mucho más densa que al inicio de la residencia, con equipos docentes específicos de medicina familiar y comunitaria dispersos en el territorio, los residentes de la segunda década han tenido también un protagonismo creativo muy importante. En sintonía con el contexto socio-histórico, han participado del compromiso asumido por la especialidad con la agenda de derechos que ocupó la escena social y política en Uruguay en los últimos años. El acceso a los derechos sexuales y reproductivos, entre ellos la gestión de riesgos y reducción de daño por interrupción voluntaria del embarazo, la atención integral a personas trans y a toda la diversidad sexual $^{13}$, así como la accesibilidad a la atención de personas que viven con VIH, han contado con labor, aporte y compromiso de docentes y residentes para incorporar estos procesos en los servicios del primer nivel. La atención a personas privadas de libertad, la perspectiva comunitaria de la salud mental, la atención a migrantes desde la perspectiva de interculturalidad y la profundización de la perspectiva de género en la atención a la salud, se encuentran hoy en la agenda de la medicina familiar y comunitaria, forman parte de su cotidianeidad y se traducen en producción académica y diseño de procesos para la atención, la gestión y el trabajo en red.

Esta producción, que surge del nuevo entramado que da soporte a la formación de residentes, es posible desde los contextos de enseñanza-aprendizaje de carácter territorial gestados a través de las Unidades Docente Asistenciales del primer nivel.

Claro que eso ocurre sin dejar de lado los problemas que tradicionalmente forman parte del trabajo médico: las enfermedades no trasmi- 
sibles y su prevalencia creciente, el cuidado de la salud en todas las etapas del ciclo vital incluida la reproducción, los cuidados paliativos, los problemas relacionados con la violencia de todo tipo, el consumo problemático de sustancias, las infecciones inespecíficas, la tuberculosis reemergente, entre otras.

Tanto para los problemas más tradicionales como para los de incorporación más reciente, es permanente el desafío de la gestión y del diseño de estrategias de accesibilidad en un Sistema Nacional Integrado de Salud que habilita y mandata a ello, pero que no siempre garantiza los recursos necesarios. La creatividad de los equipos en territorio, incluida la coordinación interinstitucional, forman parte de los procesos de micro y meso gestión que afrontan y resuelven los residentes día a día como parte de su formación.

Nuevamente ocurre que no hay una planificación ni una curricularización programada para cada paso y etapa, pero nuevamente se constata que el proceso, en su caos, resulta productivo y transformador.

\section{Crisis}

La Ley de Creación del SNIS, en su artículo 34 dice:

"El Sistema Nacional Integrado de Salud se organizará en redes por niveles de atención según las necesidades de los usuarios y la complejidad de las prestaciones. Tendrá como estrategia la atención primaria en salud y priorizará el primer nivel de atención ${ }^{11}$ ".

Con la residencia de medicina familiar y comunitaria consolidada y conquistada la legitimación académica (no sin persistencia de tensiones), se transitó un período de real jerarquización de la especialidad en el campo de la formación. El número de cargos de residencias fue creciente año a año, se aplicaron estrategias para la cobertura de vacantes ofreciendo los cargos a concursantes que hubieran aprobado el ingreso a otra especialidad básica (pediatría, ginecología, medicina interna o cirugía) y se instrumentó el ingreso a la carrera de especialista en mfyc a través del posgrado en "régimen del interior" (que ofrece horario flexible y otras ventajas para personas que viven alejadas de centros universitarios).

En Uruguay, la prueba de residencias es anual, existe un único concurso para todo el país en un mismo lugar, día y hora, pero con una prueba y un tribunal propio para cada especialidad.

La reforma de la salud no modificó las instituciones de atención a la salud que existían en Uruguay (estatales y no estatales) sino que las con- vocó a ser parte del Sistema Nacional Integrado de Salud como prestadores integrales, sujetos a las normas de funcionamiento pautadas por la Ley y administradas por el Ministerio de Salud Pública y la Junta Nacional de Salud. Antes y después de la reforma los cargos de residencia son producto de la voluntad de los efectores que los financian y proponen a la Comisión Nacional para su aprobación. La decisión final toma en cuenta también la disponibilidad de soporte académico en consulta con las respectivas Cátedras y Departamentos de la Facultad de Medicina para cada especialidad.

ASSE es el principal efector de salud del país $y$ el mayor financiador de residencias; en consecuencia, es también el ámbito más importante en formación de especialistas. Otras instituciones de asistencia médica colectiva, estatales y no estatales, ofrecen cargos de residencia en menor proporción. El concurso está a cargo de la Facultad de Medicina. Existe un Programa único para cada especialidad. Éstos son regulados por la Escuela de Graduados de la Facultad de Medicina. Cada Departamento o Cátedra de la Facultad de Medicina es responsable académico del cumplimiento del Programa durante la residencia, así como de asegurar las condiciones para la formación de los residentes.

Otra característica del Uruguay es que no se ha definido una política de recursos humanos para la salud desde la cual se decida la distribución de plazas de residencia ni el número de profesionales recomendado para cada disciplina o especialidad. El número de plazas ofrecidas y su distribución se acuerda cada año en la Comisión Nacional en base a los parámetros mencionados: las plazas ofrecidas por las instituciones prestadoras de salud y la capacidad de las Cátedras y Departamentos para garantizar la formación académica.

Algunas especialidades no quirúrgicas admiten el ingreso por la vía de posgrado sin residencia (no remunerado), tal el caso de medicina familiar y comunitaria desde el año 2003. Los aspirantes igualmente deben concursar y ganar la prueba de residencias para su admisión. Esta modalidad de ingreso representa una proporción mínima en medicina familiar y comunitaria, salvo en el año 2012 como se comentará más adelante.

La Grafica 1 muestra del número de ingresos en la especialidad desde el año 1997 a 2019 y la Gráfica 2 la variación anual de este indicador.

El pico registrado en el año 2012 corresponde a la apertura del posgrado en régimen de interior, que contó con un ingreso masivo de 23 posgrados no residentes, hecho que no tuvo continuidad en el tiempo. En el mismo año fue pico el número de plazas de residentes, que llegó a 32 . 
En la Gráfica 2 se puede ver que hasta 2012, aunque hay oscilaciones anuales en el número de ingresos, con crecimientos y descensos, predomina la tendencia al aumento. A partir de 2012 el componente negativo se hace dominante y los datos alcanzan en 2019 un ingreso de 9 residentes. Este número solo es comparable al valor del 2001 y constituye el más bajo desde entonces. A

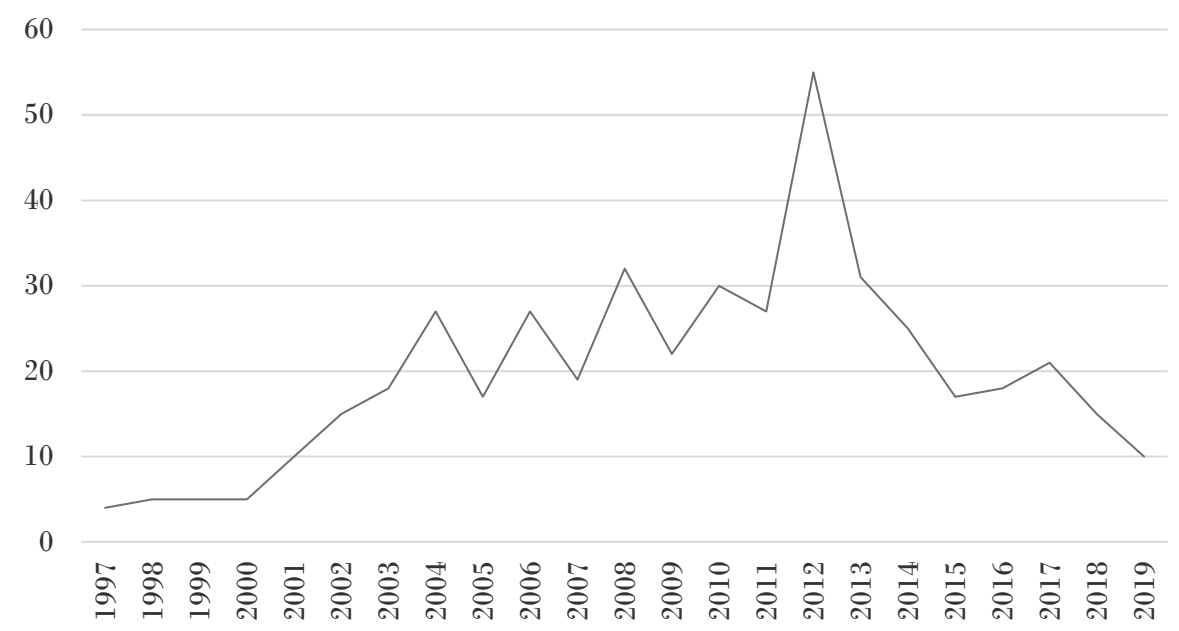

Gráfica 1. Ingresos al posgrado y residencia de medicina familiar y comunitaria desde su origen: Uruguay 19972019.

Fuente: Elaboración propia.

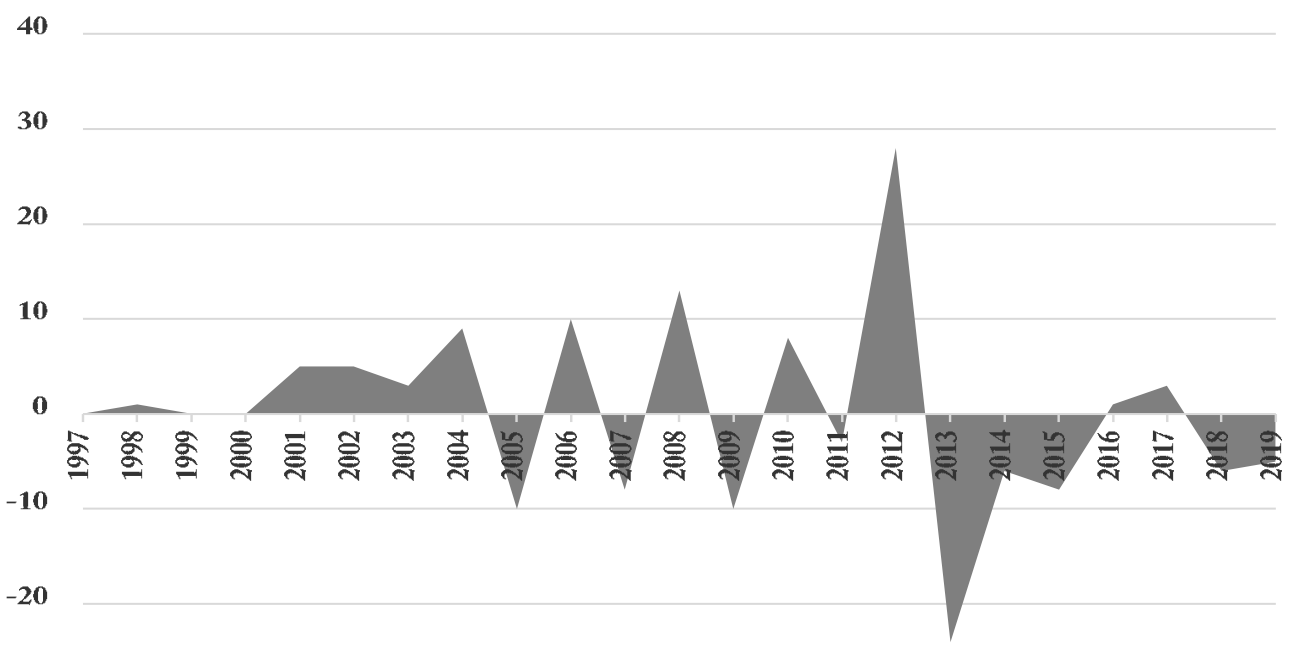

$-30$

Grafica 2. Variación anual en el número de ingresos al posgrado y la residencia de medicina familiar y comunitaria, Uruguay 1997-2019. 
diferencia de aquellos 10 cargos, que eran el cupo total para mfyc en 2001 y un cupo en ascenso luego de llamados con cuatro y cinco cargos en 1997 a 2000, en 2019 el número surge de la subocupación de plazas disponibles, y un cupo en descenso por la reiteración de la vacancia.

El descenso sostenido del ingreso con subocupación de las plazas ofrecidas, ha condicionado la pérdida de cargos en los últimos años. Como se dijo antes, las decisiones sobre la distribución son anuales y se adoptan en el ámbito de la Comisión Nacional, sin un plan estratégico rector. En esas condiciones, la reiterada subocupación de plazas es una presión negativa para el mantenimiento de un número elevado de cupos.

La búsqueda de explicaciones a esta crisis son motivo de estudio en este momento. Es conocido que no se trata de un problema aislado de Uruguay y que a pesar de los discursos y las reformas sanitarias que privilegian la atención primaria, el prestigio del trabajo en el primer nivel de atención, así como la remuneración de los profesionales, se encuentran en desventaja frente a especialistas focales y el desempeño hospitalario.

En breve estudio exploratorio realizado en el mes de agosto de 2019 se realizaron entrevistas breves a distancia a residentes, docentes y otros referentes de la medicina familiar y comunitaria; y a estudiantes avanzados de medicina y residentes de otras especialidades ${ }^{14}$. Se indagó en su visión de los motivos de este descenso en la aspiración a la residencia de medicina familiar y comunitaria. De los resultados surge, de modo no concluyente que algunos de los aspectos involucrados serían el bajo prestigio de la especialidad ("estatus del hospital"), el escaso conocimiento de la especialidad por parte de estudiantes y de la población, las condiciones de trabajo adversas: sobrecarga laboral y emocional, puestos de trabajo en contextos sociales complejos y remuneración baja comparativamente con otras especialidades.

También se identificó como influencia negativa la escasa presencia de la mfyc en los prestadores no estatales del SNIS, los cuales dan cobertura a un $60 \%$ de la población del país.

Otro aspecto, aportado particularmente por los más jóvenes, fue la preferencia por especialidades "más sencillas", "menos complejas". Pesaría en la elección la preferencia de un desempeño menos ligado a la incertidumbre.

\section{¿Alma Ata está lejos?}

Alma Ata es una ciudad. Es también una Declaración pronunciada en $1978^{15}$, en aquella ciudad de nombre peculiar, en un país que ya no existe. Pero para muchos es un faro, que no ha sido sustituido por ninguno de los documentos subsiguientes, tampoco por la más reciente Declaración de Astaná ${ }^{16}$.

Mientras nos planteamos los desafíos del futuro y las dificultades que se avizoran con este déficit de residentes que rápidamente se traducirá en déficit de especialistas y repercusiones sobre el ritmo del cambio de modelo de atención, puede resultar extraño mirar al pasado.

Si medimos el tiempo desde entonces en términos de la historia uruguaya, podemos decir que, de los 40 años que nos separan de Alma Ata, ocupamos los primeros 20 en tomar la decisión de instalar la residencia de medicina familiar y comunitaria, y los siguientes 20 años en su desarrollo. Mirado así, no parece tan lejana Alma Ata, si es que apenas ha sido suficiente el tiempo para acumular un número inicial de profesionales con la máxima competencia para el desarrollo de su estrategia. Pero ante la crisis actual cabe preguntarse si será que comenzamos a alejarnos.

La convicción de que el soporte territorial y complejo de la especialidad la coloca en el lugar cierto para los problemas de salud del siglo XXI -para acompañar, cuidar y también para investigar y gestionar-, parece chocarse con las aspiraciones de los más jóvenes que prefieren una vida profesional más estable y predecible.

La crisis a la que asistimos podría ser más profunda que el descenso de aspirantes a medicina familiar y comunitaria. Podría involucrar a la medicina, ya no solo a la especialidad. El compromiso con el tiempo histórico y el posicionamiento en la construcción de lo nuevo son condiciones inherentes al ser profesional, independiente de la profesión o especialidad. La posibilidad de estar frente a una crisis del profesionalismo en la medicina o los potenciales efectos de la mercantilización de la salud sobre la profesión, aparecen como hipótesis demasiado simples para un problema complejo, pero son ideas a tener presentes en la búsqueda de explicaciones.

Volver al pasado no parece ser la solución (aunque Alma Ata sigue vigente). Se trataría de intentar nuevos caminos, de crear, de renovar, de 
innovar. El desafío sería hacer de la medicina familiar y comunitaria una opción de vida elegible para profesionales jóvenes del siglo XXI, sin que ello signifique renunciar a Alma Ata, a ninguno de sus principios.

Nuevamente incertidumbre, la brisa que huele a cambios, la tecnología pulsando bajo nuestras almohadas, la medicina familiar y comunitaria, los sistemas de salud, los gestores que confían más en los números que en las personas, la gente con sus problemas cada vez más diversos, cada vez más complejos, el pensamiento a toda velocidad circulando en redes sociales. Todo está allí. Todo está aquí.

Repensar-nos en la profesión médica para el siglo XXI parece ser un asunto necesario, antes de retomar la cuestión de la medicina familiar y comunitaria, los bocetos antiguos y los nuevos, para preguntarnos nuevamente "cuántos kilómetros faltarán para llegar al pueblo aquel ${ }^{17 \text { " }}$.

\section{Referencias}

1. Shiva V. Monocultures of the Mind. London: Zed Books; 1993.

2. Rojas A. Policultivos de la mente. Enseñanzas del campesinado y de la agroecología para la educación en la sustentabilidad. Agroecología 2009; 4:29-38.

3. Martínez AM. Opinan Touyá, Bogliaccini, Bordes, Dibarboure y los Residentes ¿Se necesitan especialistas en Medicina Familiar? Revista Noticias (internet) 1998 [citada 2013 Oct 12]; 91. Disponible en: www. smu.org.uy/publicaciones/noticias/noticias91/familia.htm

4. Ponzo J. De Punta del Este a Barros Blancos, 2010.10.27. UDA Canelones al Este, Centro Cívico Salvador Allende [Internet Blog]. [citada 2019 Set 30]. Disponible en: http://udasalvadorallendecanelonesaleste.blogspot.com/2010/10/de-punta-del-este-barros-blancos.html

5. Asociación de Residentes de Medicina Familiar y Comunitaria del Uruguay. ARMEFYCU. La Historia de la Medicina Familiar en el Uruguay. Hacia un nuevo modelo. Revista Noticias 2005[citada 2013 Oct 12]; 130:32-33. Disponible en: www.smu.org.uy/publicaciones/noticias/noticias130/pag32-33.pdf

6. Cavallieri F, Ponzo J, Niz C. Generación de estándares de necesidad para la especialidad medicina familiar y comunitaria en el Sistema Nacional Integrado de Salud, Uruguay, 2015-2017. VI Jornadas de personal del SNIS. Octubre 2018. Sintesis de trabajos presentados. Montevideo: Ministerio de Salud Pública (publicación on line); p. 33-54. [citada 2013 Oct 12]. Disponible en: https://dgroups.org/?wqcl14hw
7. Grupo Médico Migues. Pichuaga MC, Vivas R, Díaz D, D’Agosto M, Macedo JC. La práctica médica general en un medio rural III Sobre la prevalencia e incidencia de la insuficiencia cardíaca congestiva. Rev Med Uruguay 1990; 6:9-12.

8. Dibarboure Icasuriaga H. Atención primaria de la salud: análisis de un año de trabajo médico. Montevideo: UDELAR; 1988.

9. Ponzo J. Formación de Especialistas en Medicina Familiar y Comunitaria: Desafíos y Perspectivas. V Congreso Uruguayo de Medicina Familiar. IV Congreso de Medicina Familiar del MERCOSUR, CIMF. Montevideo, 15 de diciembre de 2000.

10. Médicos Residentes de Medicina Familiar y Comunitaria de Argentina, Brasil (Río Grande do Sul), Chile, Paraguay y Uruguay. Carta de Punta del Este. Diciembre 13 de 1997. [citada 2013 Oct 12]. Disponible en: http://udasalvadorallendecanelonesaleste.blogspot. com/2010/10/de-punta-del-este-barros-blancos.html

11. Uruguay. Ley No.18.211, del 13 de diciembre de 2007. Creación del Sistema Nacional Integrado de Salud. Diario Oficial 2007; 13 dic.

12. Uruguay. Ley No 18.362 , del 15 de octubre de 2008. Rendición de cuentas y balance de ejecución presupuestal Ejercicio 2007. Diario Oficial 2008; 15 oct.

13. Uruguay. Ministerio de Salud Pública (MSP). Dirección General de la Salud. Guía clínica para la hormonización de personas trans. Montevideo: MSP-UNFPA; 2016. 
14. Ponzo J, Niz C. ¿Por qué desciende la aspiración a la residencia de medicina familiar y comunitaria? VII Jornadas de personal del SNIS. Ministerio de Salud Pública, Montevideo: 5 de setiembre de 2019. [citada 2019 Set 30]. Disponible en: www.gub.uy/ministerio-salud-publica/sites/ministerio-salud-publica/files/documentos/publicaciones/Por\%20qu\%C3\%A9\%20desciende $\% 201 \mathrm{l} \% 20$ aspiraci\%C3\%B3n $\% 20 \mathrm{a} \% 20 \mathrm{la} \% 20$ residencia $\% 20 \mathrm{de} \% 20$ medicina $\% 20$ familial $\% 20 \mathrm{y} \% 20$ comunitaria\%20-\%20Ponzo.pdf

15. Conferencia Internacional sobre Atención Primaria de Salud. Declaración de Alma-Ata. Alma-Ata, URSS, 6-12 de setiembre de 1978. [citada 2019 Set 30]. Disponible en: www.paho.org/hq/dmdocuments/2012/ Alma-Ata-1978Declaracion.pdf

16. Conferencia Mundial sobre Atención Primaria de Salud. Declaración de Astaná. Astaná (Kazajstán), 25 y 26 de octubre de 2018. [citada 2019 Set 30]. Disponible en: www.who.int/docs/default-source/primary-health/declaration/gcphc-declaration-sp.pdf

17. Benavides W, Moraes HN. La Filadelfia real. Canción. [citada 2019 Set 30]. Disponible en: www.youtube. com/watch?v $=$ oCTNmh $4 \mathrm{G} 6 \mathrm{Ok}$

Artículo presentado en 05/10/2019

Aprobado en 21/10/2019

Versión final presentada en 22/10/2019 